\title{
El efecto de la discriminación racial y étnica sobre la autoestima individual y colectiva según el fenotipo auto- reportado en migrantes colombianos en Chile
}

\author{
The effect of racial and ethnic discrimination on individual and collective \\ self-esteem according to the self-reported phenotype in Colombian migrants \\ in Chile
}

\author{
Alfonso Urzúa \\ Universidad Católica del Norte, Chile \\ Rodrigo Ferrer \\ Universidad de Tarapacá, Chile \\ Esthepany Olivares \\ Jeraldinne Rojas \\ Romy Ramírez \\ Universidad Católica del Norte, Chile
}

(Recibido: 26 de abril de 2019 (Aceptado: 20 de julio de 2019)

\begin{abstract}
Resumen
Mediante un diseño no experimental, analítico y transversal, se analizó el efecto que tiene la discriminación racial y étnica sobre la autoestima individual (AI) y colectiva (AC), según el fenotipo declarado. Para esto fueron encuestados 481 migrantes de nacionalidad colombiana ( $54 \%$ mujeres y $46 \%$ hombres), con edades entre los 18 y los 65 años, con una media de 35 años. Se aplicaron las escalas de AI de Rosenberg, de discriminación percibida de Krieger et al y la adaptación de Basabe de la escala de AC de Luthanen et al. Se observan efectos diferenciales de la discriminación étnica y/o racial sobre la AI y sobre la AC. En personas que se autoidentifican como "blancos", la percepción de discriminación racial se relaciona leve y positivamente con la AI; en quienes se perciben como "mestizos o nativos americanos", la discriminación racial tiene un efecto positivo moderado sobre la AC; en quienes se identifican como "afrodescendientes", ambas discriminaciones tienen un efecto positivo leve sobre la AI, mientras que la discriminación racial perjudicaría moderadamente la AC; y, finalmente, en quienes se identifican como "mulatos", ambas discriminaciones tendrían efectos leves o moderados positivos en los dos tipos de autoestima. Estos resultados aportan evidencia al efecto del fenotipo en la relación establecida entre la discriminación y la autoestima Palabras claves: Autoestima, autoestima colectiva, discriminación étnica, discriminación racial, migración.
\end{abstract}

Correspondencia: Dr. Alfonso Urzúa, Escuela de Psicología, Universidad Católica del Norte, Avda. Angamos 0610, Antofagasta, Chile.Email: alurzua@ucn.cl

Financiamiento: Esta publicación se deriva del proyecto FONDECYT Regular \#1180315, financiado por la Comisión Nacional de Investigación Científica y Tecnológica del Gobierno de Chile, quienes no tuvieron injerencia alguna en esta publicación. 


\begin{abstract}
Through a non-experimental, analytical and cross-sectional design, we analyze the effect that racial and ethnic discrimination has on individual (ISE) and collective (CSE) self-esteem, according to the declared phenotype. 481 Colombian migrants (54\% women and $46 \%$ men), aged between 18 and 65 (average of 35 years), were surveyed. Were applied the Rosenberg ISE scales, perceived discrimination of Krieger et al. and Basabe's adaptation of the CSE scale of Luthanen et al. Differential effects of ethnic and/or racial discrimination on ISE and on CSE are observed. In people who identify themselves as "white", the perception of racial discrimination is slightly and positively related to ISE. In those who are perceived as "mixed or Native American", racial discrimination has a moderate positive effect on CSE. In those who identify themselves as "Afro-descendants," both discriminations have a slight positive effect on ISE, while racial discrimination would moderately harm CSE. Finally, in those who identify themselves as "mulattos," both discriminations would have mild or moderate positive effects on both types of self-esteem. These results provide evidence to the effect of the phenotype on the relationship established between discrimination and self-esteem.

Keywords: Self-esteem, collective self-esteem, ethnic discrimination, racial discrimination, migration.
\end{abstract}

\title{
Introducción
}

La migración puede ser entendida como todo movimiento de personas hacia el territorio de otro Estado o dentro del mismo, sea cual fuere su tamaño, su composición o sus causas, incluyendo migración de refugiados, personas desplazadas, personas desarraigadas, migrantes económicos (OIM, 2006). Este movimiento puede deberse a motivos de supervivencia, búsqueda de nuevos territorios, factores económicos, la falta de trabajo, la violencia, el terrorismo e inseguridad, la búsqueda de bienestar social y de mejores condiciones de salud, entre otros (Vásquez, R., 2009; Guzmán, K., González, B., Rivera, M. 2014; De la Torre, 2011; Guzmán-Carrillo \& Rivera-Heredia, 2012).

El 2017, al menos 258 millones de personas vivían en un país distinto al de su nacimiento (ONU, 2018), es decir, cerca de un 3,4\% de la población mundial. Según la Organización de Naciones Unidas, al 2050 los migrantes internacionales podrían alcanzar la cifra de 405 millones de personas (OIM, 2010).

Chile no ha permanecido ajeno a esta realidad mundial. Dados los buenos resultados económicos y las condiciones de seguridad, ha sido considerado una opción para muchas personas y familias que buscan oportunidades y condiciones que no han encontrado en su país de origen (Urzúa y Cabieses, 2018). El Censo 2017 reveló que el $50.7 \%$ del total de los inmigrantes en Chile provenían de América Latina, principalmente Perú $(25,3 \%)$, Colombia $(14,2 \%)$ y Venezuela (11,2\%) (INE, 2017).

El proceso migratorio genera diversas consecuencias tanto a nivel macro-social, considerando los efectos de la migración a nivel económico, social, político y cultural, como micro-social, donde cobran relevancia las consecuencias en el plano íntimo, afectivo, emocional, entre otros. Bajo este último enfoque, se ha reportado consecuencias del migrar a nivel de la salud, el bienestar o la calidad de vida del migrante (Urzúa, Delgado, Rojas y Caqueo-Urízar, 2017; Urzúa, Heredia y Caqueo-Urízar, 2016; Urzúa, Vega, Jara et al, 2015), las cuales estarán condicionadas por variables tales como el grado de planificación del viaje, bajo qué condiciones se produce el proceso migratorio, la forma de ingreso al país receptor (vía regular o irregular), el apoyo social percibido en el país de origen y en el receptor (Urzúa, Caqueo-Urízar, Calderón y Rojas, 2017) si quien migra deja a su familia en su país de origen o si en el país receptor tiene un sitio de acogida con personas pertenecientes a su mismo país, condiciones de vida (Yáñez y Cárdenas, 2010), el nivel socioeconómico, nivel cultural, expectativas en el país de llegada, idioma y grado de similitud entre la cultura de acogida y la de origen que son variables que si bien pertenecen más a un enfoque macro social, tienen directa repercusión a nivel personal, ya que la persona va modulando estos nuevos cambios (Valiente, Sandín, Chorot, Santed y González de Rivera, 1996).

La migración tiene repercusiones en la salud de los inmigrantes dada su estrecha relación con potenciales eventos estresantes, tales como el asentamiento en el entorno cultural de acogida, diferencias de idioma y costumbres, discriminación y actitudes hostiles de la sociedad de acogida (Urzúa, Basabe, Pizarro, Ferrer, 2017; Ward, Bochner, y Furnham, 2001). Estos efectos podrían estar mediados por distintos factores puesto que existen evidencias de que las personas poseen elementos que les permiten adaptarse a los nuevos contextos sociales de 
acogida o recepción (Mascie-Taylor \& Little, 2004, Lueck \& Wilson, 2011), como por ejemplo las estrategias de aculturación (Urzúa, Ferrer, Canales et al, 2017). El periodo de adaptación dependerá en gran medida de la personalidad del individuo, de las razones que lo llevaron a migrar, de los recursos de los que disponga para hacer frente a la situación, así como del apoyo que le brinde la nueva sociedad a la que ha llegado facilitando la integración de la cultura de acogida y el mantenimiento de su propia identidad cultural (Arenas y Urzúa, 2016; Bhugra, 2004; Pinillos, 2012). Las consecuencias más comunes del proceso migratorio se relacionan con problemas de salud física y mental, en el sentido económico con la escasez de recursos para satisfacer sus necesidades básicas, pobreza y condiciones de vida inhumanas, desempleo, marginación, invisibilización, discriminación y explotación (Valiente et al., 1996, Zarza y Sobrino, 2007; Urzúa, Boudon y Caqueo-Urizar, 2017), variables que influyen directamente en la calidad de vida y bienestar de cada sujeto.

Uno de los principales factores reportados que impacta tanto en la salud como el bienestar es la discriminación, la cual afecta de manera negativa a la población inmigrante (Lewis, Cogburn \& Williams, 2015; Zlobina, 2004). Las revisiones sobre discriminación y salud han documentado asociaciones bastante fuertes y consistentes entre las experiencias autoinformadas de discriminación y una variedad de indicadores de salud mental y bienestar psicológico (Paradies 2006, Pascoe y Richman 2009; Schmitt, Branscombe, Postmes y García, 2014; Williams y Mohammed 2009; Jasinskaja-Lahti, Liebkind, Jaakkola y Reuter, 2006; Schmitt y Branscombe, 2002).

La Convención Internacional sobre la Eliminación de todas las Formas de Discriminación Racial definió la discriminación como "todas distinción, exclusión, restricción o preferencia basada en motivos de raza, color, linaje $\mathrm{u}$ origen nacional o étnico que tenga por objeto o por resultado anular o menoscabar el reconocimiento, goce o ejercicio, en condiciones de igualdad, de los derechos humanos o libertades fundamentales en las esferas política, económica, social, cultural o en cualquier otra esfera de la vida pública" (Aguilar, 2014). La perspectiva psicológica conceptualiza la discriminación como un tipo de estrés, es decir, como un evento ambiental (un evento estresante) que requiere un importante ajuste psico-social (Brondolo, Rieppi, Kelly, \& Gerin, 2003; Brondolo, Kelly, Coakley et al., 2005; Clark, Anderson, Clark \& Williams, 1999; Contrada, Ashmore, Gary, Coups, Egeth, Sewell, et al.,2001; Myer, 2003). Aun cuando existen diversas formas en las que la discriminación puede ser ejercida, en este estudio consideraremos únicamente la discriminación étnica y racial, puesto que son las que se ejercen con mayor frecuencia en población migrante, haciendo referencia, en el caso de la discriminación racial, a todo trato diferenciado por motivos de raza o color, el trato diferenciado no tiene una justificación objetiva y razonable si no persigue un objetivo legítimo o si no existe una relación razonable de proporcionalidad entre los medios empleados y el objetivo que se pretende alcanzar (Campal, 2014). Por otro lado, la discriminación étnica es entendida como procesos de diferenciación y separación. Implica hablar de situaciones de desigualdad y de exclusión producto de la xenofobia, el odio al extranjero, con manifestaciones que van desde el rechazo más o menos manifiesto, el desprecio y las amenazas, hasta las agresiones y asesinatos. (Velásquez, 2016).

Diversos reportes han mostrado que el impacto de la discriminación depende, entre otras cosas, del significado particular de pertenecer al grupo en cuestión (Mckoy \& Mayor, 2003), de las formas de vinculación con otros grupos sociales (Noh \& Kaspar, 2003), de las señales (amenazantes) que ofrecen los contextos cotidianos de interacción (Steele \& Aronson, 1995), de las posiciones de los grupos en las relaciones de poder (Schmitt y Branscombe, 2002) y en particular, de las formas en que las personas interpretan las causas del trato injusto (Foster, 2000; Hansen \& Sassenberg, 2006).

Una de las líneas que se ha desarrollado tiene como objetivo analizar la relación existente entre la autoestima y la discriminación. Se ha reportado que la autoestima pudiese tener un efecto mediador entre la discriminación y diversas variables vinculadas al bienestar, tales como el efecto de la discriminación racial sobre la salud mental (Tynes, Umaña-Taylor, Lin y Anderson, 2012), los rasgos de personalidad y estima corporal (Skorek, Song, Dunham, 2014), el grado de perseverancia y entusiasmo al enfrentar situaciones particulares y la satisfacción con la vida (Li, Fang, Wang, Sun, \& Cheng, 2018), el apoyo social y la satisfacción con la vida (Kong y You, 2013), el apoyo social y bienestar subjetivo (Kong, Zhao y You, 2013), la inteligencia emocional y la satisfacción con la vida (Kong, Zhao, y You, 2012) o el auto-estigma y la calidad de vida (Huang, Chen Pakpour y Lin, 2018), entre otros. Pese a esto, no existe abundante literatura que profundice la estrecha relación que pareciera existir entre la discriminación y la autoestima. 
La autoestima individual (AI) puede ser definida como el sentimiento que uno tiene hacia sí mismo, el cual puede ser positivo o negativo, y se construye por medio de la evaluación de las propias características personales (Rosenberg, 1965). La autoestima colectiva (AC) se refiere a la evaluación que hace el propio individuo y a la percepción de la evaluación que hacen otros acerca de esos grupos (Tajfel y Turner, 1986).

Se ha reportado que la AI se ve afectada negativamente por la percepción de haber experimentado alguna situación de discriminación (Branscombe, Schmitt y Harvey,1999; Bourguignon, Seron, Yzerbyt y Herman, 2006; Schmitt \& Branscombe, 2002), afectando el bienestar psicológico de las personas dada la estrecha relación entre las satisfacciones que tienen las personas con sus propias vidas y sus formas de percibir, pensar, actuar y sentir (Abello, Amarís, Blanco et al, 2008).

En estudios previos en Chile, se ha analizado el papel de la AI como variable mediadora entre la discriminación y la salud mental (Urzúa, Cabrera, Calderón y Caqueo-Urizar, 2019) y entre la discriminación y el bienestar psicológico (Urzúa, Ferrer, Godoy, Leppes, Trujillo, Osorio, Caqueo-Urízar, 2018), donde se encontró un efecto mediador parcial de la AI sobre esta última relación. Pese a esto, no se ha profundizado en la relación que pudiese existir entre la discriminación y la autoestima, considerando que existe más de un tipo de discriminación y más de un tipo de autoestima, a la vez que esta relación pudiese presentarse de manera diferente dependiendo de terceros factores, como sería en el caso particular de esta investigación, el fenotipo auto-reportado.

En este contexto, la presente investigación profundiza dichos hallazgos, teniendo como objetivo evaluar la relación existente entre dos tipos de discriminación asociados con mayor frecuencia al migrante (étnica y racial), tanto sobre la AI como sobre la AC, hipotetizando que estas relaciones diferirán dependiendo del fenotipo declarado por la persona, considerando que existe evidencia que reporta un comportamiento diferencial de la autoestima dependiendo de la adscripción racial.

\section{Método}

\section{Participantes}

Fueron encuestadas 481 personas, todos inmigrantes de nacionalidad colombiana, mayores de 18 años, residentes en la ciudad de Antofagasta, Chile, sin limitaciones respecto al tiempo establecido desde su llegada. La elección de los participantes se realizó mediante un muestreo no probabilístico, intencionado, en función de su accesibilidad y cumpliendo con los criterios de inclusión antes mencionados, mediante un muestreo combinado de Bola de nieve (Goodman, 1961) y un muestreo dirigido por los entrevistados, con el fin de intentar equiparar cuotas por sexo.

Del total de entrevistados, un $46 \%$ corresponde a hombres y el $54 \%$ a mujeres. La edad de los participantes osciló entre los 18 y los 65 años. De los encuestados se auto identificó un 26,6\% (n=128) como "blancos", siendo $44,5 \%$ hombres ( $n=58)$ y $55,1 \%(n=70)$ mujeres; $26,4 \%(n=127)$ como "mestizos o nativos americanos", siendo $44,1 \%$ hombres $(\mathrm{n}=56)$ y $55,9 \%(\mathrm{n}=71)$ mujeres; $30,8 \%(\mathrm{n}=159)$ como "afrodescendientes", siendo $44,7 \%$ hombres $(\mathrm{n}=71)$ y $55,3 \%(\mathrm{n}=88)$ mujeres; y $13.9 \%(\mathrm{n}=67)$ como "mulatos", siendo $49,3 \%$ hombres $(\mathrm{n}=33)$ y $50.7 \%(\mathrm{n}=34)$ mujeres. No se observó interacciones $(\mathrm{p}<, 05)$ entre auto identificación fenotípica y sexo biológico $\left(\chi_{\mathrm{GL}=3}^{2}=0.531 ; \mathrm{p}=.91\right)$.

\section{Instrumentos}

\section{Discriminación Percibida}

Se utilizó la escala de experiencias de discriminación propuesta por Krieger, Smith, Naishaham, Hartman y Barbeau et al (2005), la cual pretende medir la percepción que tienen los sujetos respectos a las situaciones que han experimentado vinculadas a la discriminación. Para efectos de medir la discriminación reciente, se utilizó el módulo de percepción de trato injusto cotidiano. 


\section{Autoestima}

Para medir la autoestima individual, se utilizó la Escala de Autoestima de Rosenberg (1965), compuesta por 10 ítems respecto de los sentimientos de satisfacción que cada persona tiene de sí mismo que permite obtener entre los 10 puntos (baja autoestima) y los 40 (alta autoestima). Las características psicométricas de esta escala han sido ampliamente estudiadas en su versión original americana, así como en otros idiomas (Kernis y Grannemann, 1991; Roberts y Monroe, 1992; Rojas-Barahona, Zegers, \& Förster, 2009), así como en población colombiana (Gómez-Lugo, Espada, Morales Marchal-Bertrand, Soler, Vallejo-Medina, 2016).

Para la autoestima colectiva, se utilizó la versión adaptada de Basabe (Basabe, Páez, Aierdi y JiménezAristizábal, 2009) de la escala propuesta por Luthanen y Crocker (1992).

\section{Fenotipo}

Se le solicitó a cada participante que se auto adscribiese a una de las siguientes categorías: 1) Blanco-caucásico; 2) De pueblos indígenas (originarios) sudamericanos (wayuu, senú, pasto, coyaima, quechua, aymará, amazónicos, etc); 3) Mestizo: mezcla de blanco y pueblos indígenas originarios; 4) Negro (afrodescendiente); 5) Mulato: mezcla de negro y blanco

\section{Análisis estadístico}

Se probaron y depuraron los modelos de medida, estimando fiabilidades mediante coeficientes omega de McDonald y alfa de Cronbach, así como, siguiendo recomendaciones de diversos autores sobre el tratamiento factorial de variables ordinales (Garrido, Abad, y Ponsoda, 2011), análisis factoriales confirmatorios a partir de la matriz de variables policóricas, utilizando el método de estimación robust weighted least squares (WLSMV), el cual es robusto con variables discretas no normales (Nylund, Asparouhov y Muthen, 2007). Todas las depuraciones realizadas fueron acompañadas de análisis de ítems.

Finalmente, utilizando el mismo método de estimación (WLSMV) y las matrices de correlaciones policóricas, se estimaron los tamaños del efecto y el ajuste global de los modelos de efectos directos de la discriminación racial percibida, diferenciadas por grupos, sobre la autoestima colectiva, mediante modelos de ecuaciones estructurales. Finalmente, se realizaron comparaciones de coeficientes de regresión, mediante la transformación Fisheriana de $\mathrm{r}$ a $\mathrm{z}$, la cual ha evidenciado ser una estrategia útil para la comparación entre dos grupos, con independencia de las características distribucionales de origen y el método de estimación utilizado (Myers \& Sirois, 2004). Todos los análisis con variables latentes se efectuaron con la versión 8.2 del programa MPLUS, mientras que los análisis de fiabilidad se hicieron con la versión 0.9.5.11 del programa jamovi.

\section{Resultados}

\section{Modelos de Medida}

La tabla 1 presenta el ajuste de los modelos de media de las tres escalas utilizadas. Sin embargo, algunas relaciones entre observaciones se encontraban insuficientemente representadas por los modelos iniciales, observándose niveles de ajuste inferiores a los estándares recomendados en la literatura (i.e. CFI $>, 95$; TLI $>, 95$; RMSEA $<, 08$ ) (Schreiber, Nora, Stage, Barlow y King, 2006). Debido a esto, se procedió a depurar iterativamente los modelos iniciales, reduciéndose las escalas utilizadas para las tres escalas utilizadas en el modelo. 
Tabla 1. Indicadores de ajuste global de los modelos de medidas en la muestra total.

\begin{tabular}{|c|c|c|c|c|c|c|c|c|c|c|}
\hline \multirow{2}{*}{$\begin{array}{l}\text { Modelo de } \\
\text { medida }\end{array}$} & \multirow[t]{2}{*}{$\mathbf{N}^{\circ} \operatorname{Par}$} & \multirow[t]{2}{*}{$\chi^{2}$} & \multirow[t]{2}{*}{ DF } & \multirow[t]{2}{*}{$\mathbf{p}$} & \multirow[t]{2}{*}{ CFI } & \multirow[t]{2}{*}{ TLI } & \multirow[t]{2}{*}{ RMSEA } & \multicolumn{2}{|c|}{$\begin{array}{l}\text { RMSEA CI } \\
\mathbf{9 0 \%}\end{array}$} & \multirow[t]{2}{*}{ SRMR } \\
\hline & & & & & & & & Low & Upp & \\
\hline $\begin{array}{l}\text { Discriminación } \\
(20 \text { ítems) })_{1}\end{array}$ & 91 & 991.070 & 159 & .000 & .948 & .938 & .103 & .096 & .109 & .070 \\
\hline $\begin{array}{l}\text { Discriminación } \\
(16 \text { ítems) } \\
1,2\end{array}$ & 75 & 335.191 & 93 & .000 & .979 & .973 & .072 & .064 & .081 & .045 \\
\hline AI (10 ítems) & 41 & 1169 & 35 & .000 & .817 & .765 & .255 & .243 & .268 & .154 \\
\hline AI (5 ítems) ${ }_{3}$ & 20 & 11.918 & 3 & .007 & .998 & .994 & .077 & .035 & .126 & .008 \\
\hline AC (6 ítems) & 42 & 185.094 & 9 & .000 & .930 & .883 & .198 & .174 & .224 & .059 \\
\hline $\mathrm{AC}(3 \text { items })_{4}$ & 21 & .000 & 0 & .000 & 1.00 & 1.00 & .000 & .000 & .000 & .001 \\
\hline
\end{tabular}

Covariación de ítems espejo (ítems que poseen una misma redacción, pero cambia el objetivo de referencia); 2 eliminación de los ítems 2 y 3 de las dimensiones discriminación racial y étnica, por presentar redundancias semánticas y estadísticas severas, y covariación de ítems 8,9 y 10 de la dimensión discriminación racial, por presentar redundancias internas menores; ${ }_{3}$ eliminación de ítems 3, 5, 8, 9 y 10, los que correspondían a los ítems inversos de la escala, ya que, por factores de método, poseen redundancias severas y saturaciones factoriales de baja representatividad $(\lambda<.4)$, incluso ante la adición de un factor de método; 4 modelo saturado, pero con saturaciones factoriales grandes $(\lambda>.7)$, eliminándose los ítems 3,4 y 6 , por presentar saturaciones factoriales de baja representatividad $(\lambda<.4)$.

Finalmente, se presentan las estimaciones de fiabilidad de cada instrumento y subdimensión, a partir de los modelos depurados (tabla 2).

Tabla 2. Coeficientes omega y alfa para cada dimensión

\begin{tabular}{lcccc}
\hline & $\begin{array}{c}\text { Autoestima } \\
\mathbf{( 5} \text { ítems })\end{array}$ & $\begin{array}{c}\text { Autoestima Colectiva } \\
\mathbf{( 3 ~} \text { ítems) }\end{array}$ & \multicolumn{2}{c}{ Discriminación percibida } \\
\cline { 4 - 5 } & .914 & .853 & $\begin{array}{c}\text { Étnica } \\
\mathbf{( 8} \text { ítems })\end{array}$ & $\begin{array}{c}\text { Racial } \\
(\mathbf{8} \text { ítems })\end{array}$ \\
\hline Cronbach's $\alpha$ & .915 & .864 & .890 & .918 \\
\hline McDonald's $\omega$ & & .891 & .919 \\
\hline
\end{tabular}

Se observa que la mayoría de las dimensiones estudiadas reportan estimaciones de fiabilidad que presentan niveles óptimos de consistencia interna $(\omega>.90)$ o, al menos, adecuados $(\omega>.80)$.

A partir de los modelos de medida depurados, se contrastó el modelo hipotetizado en el estudio, diferenciado por discriminación racial (figura 1) y étnica (figura 2), tanto a nivel general como diferenciado para cada grupo étnico. Los indicadores de ajuste de los modelos generales, no diferenciados por grupos, se presentan en la tabla 3. 


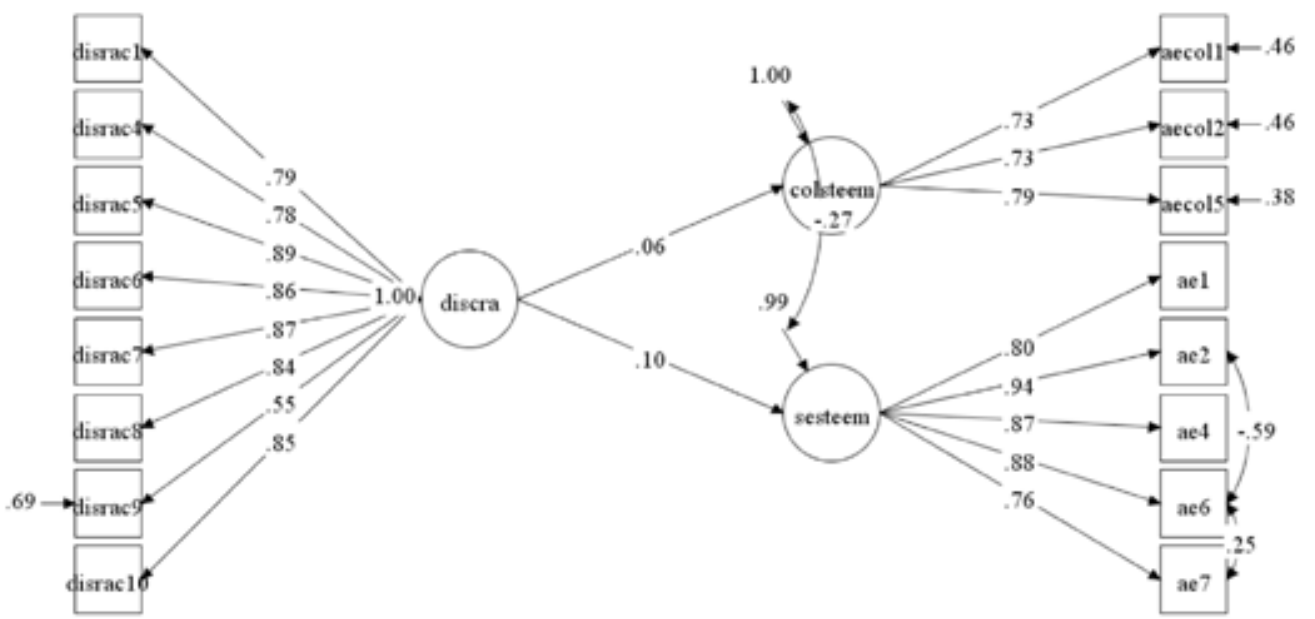

Figura 1. Representación del modelo general $(\mathrm{n}=481)$, con saturaciones estandarizadas, del modelo basado en discriminación racial.

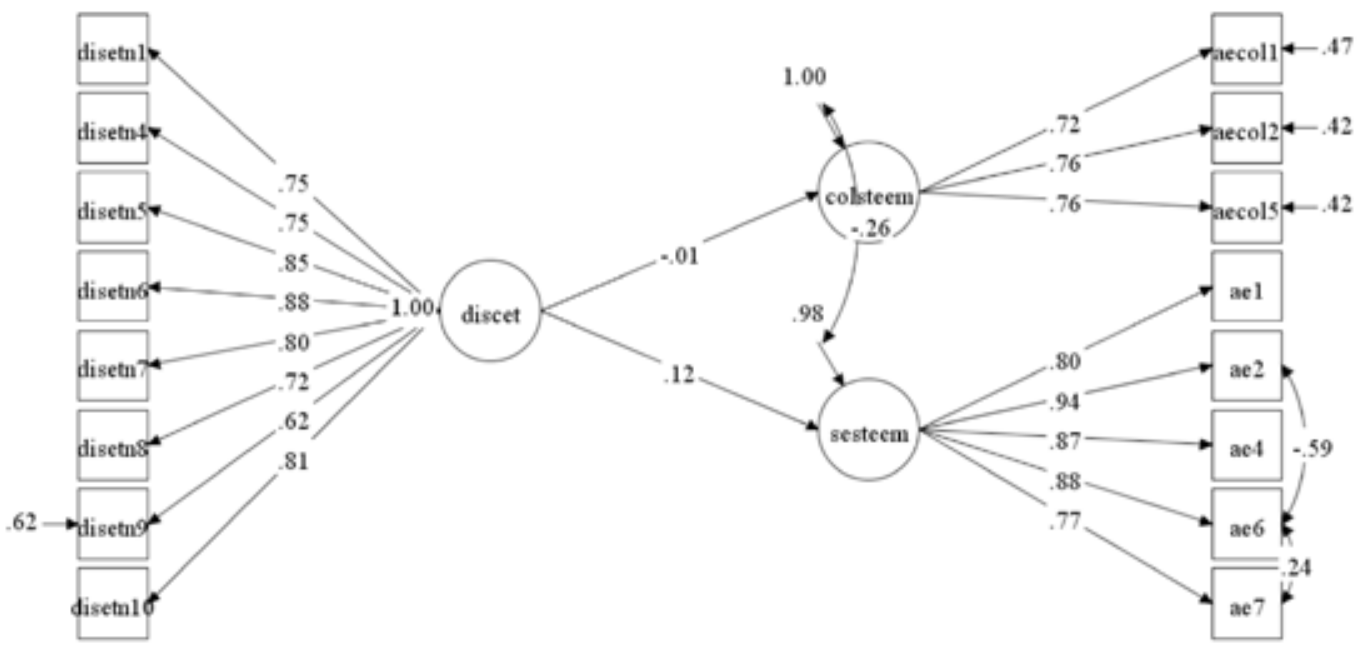

Figura 2. Representación del modelo general $(\mathrm{n}=481)$, con saturaciones estandarizadas, del modelo basado en discriminación étnica.

Tabla 3. Indicadores de ajuste global de los modelos generales $(n=481)$

\begin{tabular}{cccccccccccc}
\hline & $\mathrm{N}^{\circ}$ Par & $\chi 2$ & DF & $\mathrm{p}$ & CFI & TLI & RMSEA & \multicolumn{2}{r}{ RMSEA CI } & SRMR \\
\hline $\begin{array}{c}\text { Discriminación } \\
\text { racial }\end{array}$ & 65 & 171.912 & 99 & .000 & .992 & .990 & .040 & .030 & .050 & .040 \\
\hline $\begin{array}{c}\text { Discriminación } \\
\text { étnica }\end{array}$ & 65 & 217.644 & 99 & .000 & .983 & .980 & .051 & .042 & .060 & .047 \\
\hline
\end{tabular}

Ambos modelos generales $(\mathrm{n}=481)$ presentaron adecuados niveles de ajuste, acorde con los estándares recomendados en la literatura (i.e. CFI $>, 95$; TLI $>, 95$; RMSEA $<$,08) (Schreiber, Nora, Stage, Barlow y King, 2006), evidenciando ser una representación suficiente de las relaciones observadas. No obstante, en los modelos 
generales, solo se observa un efecto poblacional distinto de cero en el caso de la percepción de discriminación étnica sobre la autoestima individual ( $\mathrm{p}>.05)$. Las estimaciones estandarizadas de los efectos directos de la discriminación percibida, tanto racial como étnica, diferenciados por grupo, se representan en la tabla 4.

Tabla 4. Estimaciones de efectos estandarizados, diferenciados por grupo.

\begin{tabular}{|c|c|c|c|}
\hline Grupo & $\begin{array}{c}\text { Discriminación } \\
\text { percibida }\end{array}$ & Autoestima & $\begin{array}{c}\text { Autoestima } \\
\text { colectiva }\end{array}$ \\
\hline \multirow{2}{*}{ Modelo general $(n=481)$} & Racial & .097 & .059 \\
\hline & Étnica & $.125^{*}$ & -.011 \\
\hline \multirow{2}{*}{ “Blancos" (n=127) } & Racial & $.267 * *$ & -.181 \\
\hline & Étnica & .092 & -.102 \\
\hline \multirow{2}{*}{ “Mestizos y nativos americanos" $(n=128)$} & Racial & .031 & $.370^{*}$ \\
\hline & Étnica & .015 & .011 \\
\hline \multirow{2}{*}{ “Afrodescendientes" (n=159) } & Racial & $.300 *$ & $-.335^{*}$ \\
\hline & Étnica & $.274 * *$ & -.085 \\
\hline \multirow{2}{*}{ "Mulatos" $(n=67)$} & Racial & $.441^{*}$ & $.363 *$ \\
\hline & Étnica & $.357 * *$ & $.289 * *$ \\
\hline
\end{tabular}

Se observan efectos diferenciales del tipo de discriminación entre grupos, observándose algunos efectos (b>.30; Cohen,1988) y leves (b>.10; Cohen, 1988) de la discriminación étnica y/o racial, sobre la AI y sobre la AC. Es así como, en apariencia: en personas que se autoidentifican como "blancos", la percepción de discriminación racial se relaciona leve y positivamente con el autoestima individual; en quienes se perciben como "mestizos o nativos americanos", la discriminación racial tiene un efecto positivo moderado sobre el autoestima colectiva; en quienes se identifican como "afrodescendientes", tanto la discriminación étnica como racial tienen un efecto positivo leve sobre el autoestima individual, mientras que la discriminación racial perjudicaría moderadamente el autoestima colectiva; $y$, finalmente, en quienes se identifican como "mulatos" tanto la discriminación étnica como la racial tendrían un efectos leves o moderados positivos, tanto sobre el autoestima colectiva como sobre el autoestima individual.

Finalmente, para evaluar si los efectos observados son diferentes entre grupos, se compararon los coeficientes de regresión latente, de acuerdo con la probabilidad calculada a partir de la transformación de Fisher de r a z. Los resultados se presentan en la tabla 5 . 
Tabla 5. Estimaciones de probabilidad de diferencias entre grupos a dos colas.

\begin{tabular}{|c|c|c|c|c|}
\hline Grupo & $\begin{array}{l}\text { Discriminación } \\
\text { percibida }\end{array}$ & "Blancos" & $\begin{array}{c}\text { "Mestizos y nativos } \\
\text { americanos" }\end{array}$ & "Afrodescendientes" \\
\hline \multicolumn{5}{|c|}{ Autoestima individual } \\
\hline \multirow{2}{*}{ "Blancos" (n=127) } & Racial & - & & \\
\hline & Étnica & - & & \\
\hline \multirow{2}{*}{$\begin{array}{l}\text { "Mestizos y nativos americanos" } \\
(\mathrm{n}=\mathbf{1 2 8})\end{array}$} & Racial & .056 & - & \\
\hline & Étnica & .541 & - & \\
\hline \multirow{2}{*}{ “Afrodescendientes" $(n=159)$} & Racial & .764 & $.020 *$ & - \\
\hline & Étnica & .116 & $.026^{*}$ & - \\
\hline \multirow{2}{*}{ "Mulatos" (n=67) } & Racial & .193 & $.004 * *$ & .271 \\
\hline & Étnica & .067 & $.019^{*}$ & .535 \\
\hline \multicolumn{5}{|c|}{ Autoestima colectiva } \\
\hline \multirow{2}{*}{ “Blancos" (n=127) } & Racial & - & & \\
\hline & Étnica & - & & \\
\hline \multirow{2}{*}{$\begin{array}{l}\text { "Mestizos y nativos americanos" } \\
(\mathrm{n}=128)\end{array}$} & Racial & $.000 * *$ & - & \\
\hline & Étnica & .373 & - & \\
\hline \multirow{2}{*}{ “Afrodescendientes" $(n=159)$} & Racial & .167 & $.000 * *$ & - \\
\hline & Étnica & .888 & .423 & - \\
\hline \multirow{2}{*}{ "Mulatos" (n=67) } & Racial & $.000 * *$ & .960 & $.000 * *$ \\
\hline & Étnica & $.009 * *$ & .062 & $.009 * *$ \\
\hline
\end{tabular}

$* \mathrm{p}<.05 * * \mathrm{p}<.01$

En el caso de la autoestima individual, se aprecia que, tanto en la discriminación étnica como en la discriminación racial, existen efectos diferenciales en los "mestizos o nativos americanos" respecto a "afrodescendientes" y "mulatos". Finalmente, en el caso de la autoestima colectiva, se observan efectos diferenciales la discriminación racial en "blancos" y "afrodescendientes", respecto a "mestizos o nativos americanos" y "mulatos"; así como efectos diferenciales de la discriminación étnica en "afrodescendientes" respecto a "mulatos".

\section{Discusión}

El objetivo de la investigación fue analizar el efecto de la discriminación tanto étnica como racial sobre la AI y la $\mathrm{AC}$, a la vez que examinar cómo este podría variar según el fenotipo auto-reportado por las personas inmigrantes.

Se encuentra que en la población auto-categorizada como "blanca", la discriminación racial tiene un efecto moderado y positivo sobre la AI, es decir, mientras mayor sea la discriminación ejercida en dicha población, mayores serán sus niveles de AI. Esto podría ser producto de que cuando las personas evalúan la discriminación personal y grupal, utilizan diferentes tipos de comparación (Postmes, Branscombe, Spears y Young, 1999), en este caso en particular, el discrimen podría ser positivo para la gente que se percibe "blanca", aun cuando también es posible que la discriminación no sea percibida por algunas personas, como una forma de proteger su autoestima de los efectos negativos del fracaso (Crocker y Major, 1989).

Con relación a los auto-categorizados como "mestizos", se observa que la discriminación étnica tiene grandes y negativos efectos sobre la AC, la que se verá disminuida toda vez que el grupo se sienta discriminado por razones étnicas por parte de otros. Se ha demostrado que la autoestima se ve afectada negativamente por la percepción de discriminación (Branscombe et al., 1999; Bourguignon, Seron, Yzerbyt y Herman, 2006; Schmitt, Branscombe et al., 2002). No obstante, la discriminación también podría conducir a la marginación, que podría estar asociada con un bajo nivel de autoestima (Urzúa, Ferrer, Godoy et al, 2018). Este escenario de someti- 
miento y descalificaciones tiene incidencias con marcada regularidad en relación con conflictos de identidad, desánimo, depresión y fuerte deterioro de la autoestima. La evaluación social de los aspectos colectivos del sí, o la estima que la sociedad asigna a los grupos de pertenencia de la persona son otra fuente de autoestima. Las relaciones laborales, por ejemplo, muy bien pueden ser uno de los contextos del mundo real más importantes y bien documentados con respecto a la discriminación intergrupal que, sobre todo, ocurre en relación con el origen étnico y el sexo como un problema importante (Lönnqvist, Hennig-Schmidt y Walkowitz, 2015).

En este mismo grupo, paradójicamente los efectos de la discriminación racial en la AC son grandes y positivos, lo cual evidencia que el grupo aumentará su autoestima a nivel colectivo en función de la discriminación racial percibida y/o ejercida sobre ellos. Estudios señalan que, un factor en la alta autoestima de personas de raza negra pueden ser las propiedades de autoprotección que ofrece la membresía en un grupo estigmatizado o en desventaja. Desde hace muchos años se ha sugerido que la membresía en grupos minoritarios raciales puede proteger o amortiguar la autoestima (Crocker \& Major, 1989; McCarthy y Yancey, 1971; Rowley, Sellers, Chavous y Smith, 1998). Por ejemplo, las minorías raciales pueden comparar sus resultados de forma selectiva con los de otras personas con desventajas similares (Broman, Neighbours \& Jackson, 1988; Crocker \& Major, 1989; Major, 1994; Major, Sciacchitano \& Crocker, 1993; Pettigrew, 1967; Porter \& Washington, 1979), atribuyen fallas o rechazos personales a los prejuicios (Crocker, Voelkl, Testa, \& Major, 1991; Dion, 1986), o devalue los dominios en los que su grupo tiene una calificación pobre, valorando selectivamente los dominios en los que su grupo tiene un buen desempeño (Crocker \& Major, 1989; Heiss \& Owens, 1972; Schmader \& Major, 1999; Steele, 1997). De acuerdo con este punto de vista, la investigación sobre minorías raciales con circunstancias históricas bastante diferentes ha encontrado que tienen una autoestima relativamente alta.

Por otro lado, los efectos de la discriminación étnica en población afrodescendiente sobre la autoestima individual serán leves y positivos, indicando un aumento de la autoestima en la medida en que se ejerza mayor discriminación étnica. Se ha encontrado que hay miembros de grupos estigmatizados que, cuando reconocen haber sido víctimas de discriminación, pueden aumentar la identificación con su grupo étnico como parte de sus estrategias de afrontamiento, contrarrestando el impacto negativo que la discriminación étnica tiene en el valor propio y en la autoestima individual. Por lo tanto, el tipo de relación entre la discriminación y la autoestima dependerá de una serie de factores, que confirman que a veces la discriminación hacia un grupo minoritario fortalecerá la identidad étnica, lo que aumentará la autoestima de sus miembros. En este contexto, Bourguignon et al. (2006) señala que la discriminación grupal, al hacer sentir menos solos a los miembros de estas minorías, produce un efecto positivo en la autoestima.

Respecto a los efectos de la discriminación racial sobre la autoestima colectiva en la población afrodescendiente, se puede señalar que, estos se presentan de manera leve y negativa, indicando menores niveles de autoestima colectiva respecto a la discriminación racial percibida. Desde la perspectiva de la estigmatización, se sostiene que algunos miembros de los grupos minoritarios raciales, como los afroamericanos, desarrollarían una baja autoestima como consecuencia de interiorizar las opiniones negativas del grupo cultural dominante (Crocker y Major, 1989; Twenge y Crocker, 2002). Las teorías que sostienen que la autoestima se desarrolla a partir de las opiniones de otras personas sobre el yo (Cooley, 1902; Mead, 1934) llevaron a la hipótesis de que los negros, como objetivos del prejuicio y la discriminación, deberían sufrir de baja autoestima. Aunque Cooley (1902) originalmente propuso que solo las opiniones de otros "significativos" se incorporarán al concepto de sí mismo, Mead (1934) sugirió que las opiniones de otros "generalizados", o el contexto sociocultural completo de la persona, pueden incorporarse en el autoconcepto. Por lo tanto, Jones (1999) ha argumentado que un aspecto importante del racismo es el racismo cultural, en el que el grupo dominante o más poderoso define lo que se valora en una cultura, así como las formas específicas que pueden adoptar esas características valiosas. Debido a que estas definiciones casi siempre favorecen los atributos del grupo dominante, el resultado es una devaluación generalizada de los grupos minoritarios subordinados y según esta hipótesis, la internalización del estigma conduce a una baja autoestima entre los grupos minoritarios.

Finalmente, con relación a los Mulatos, se observan efectos positivos y moderados de la discriminación étnica sobre la autoestima colectiva, reportando efectos directos entre las variables señaladas, es decir, su autoestima colectiva aumentará a mayor discriminación étnica. Respecto a este punto, esta respuesta podría explicarse producto de una estrategia de rechazo-identificación, planteada por Branscombe, Schmitt y Harvey (1999). Estos señalaron que la identificación puede servir de amortiguador entre la discriminación y la autoestima. Al mismo tiempo, los teóricos de la identidad social (Ellemers, Wilke y Van Knippenberg, 1993; Tajfel \& Turner, 
1986), han propuesto que las personas discriminadas tienden a reaccionar al comportamiento injusto mediante sentimientos de identificación con su grupo y haciendo que esta tenga un impacto positivo en su autoestima como grupo. En conjunto, estas ideas sugieren que el impacto de la discriminación es probablemente amortiguado por una mayor identificación, contrarrestando los efectos nocivos de la discriminación en la autoestima.

Estos análisis indican que los efectos de las discriminaciones racial y étnica sobre la AI y la AC no son uniformes: los efectos difieren considerablemente entre los grupos, no sólo en magnitud sino también en la dirección. Esto podría explicarse por las diferencias individuales-culturales asociadas a cada fenotipo.

Respecto a lo anterior, se puede señalar que existen diferencias significativas de discriminación étnica entre los grupos mestizos y blancos respecto de mulatos y afrodescendientes en cuanto a la autoestima individual, donde estos últimos presentan mayor percepción de discriminación étnica a excepción de los mulatos y mestizos, puesto los mestizos presentan mayor percepción de discriminación étnica que los mulatos. En relación a la autoestima individual, se observa que blancos y mestizos presentan mayores niveles que afrodescendientes y mulatos, salvo afrodescendientes y blancos ya que este último presenta menores niveles de autoestima individual que los afrodescendientes toda vez que perciben discriminación por motivos étnicos.

Por otro lado, se observa que en cuanto a la discriminación racial, existen diferencias significativas entre los grupos de blancos respecto de mestizos y afrodescendientes, donde estos últimos presentan mayor percepción de discriminación racial que los blancos, al igual que mayores niveles de autoestima individual.

En cuanto a la autoestima colectiva, es posible señalar que todos los grupos difieren en los efectos de la discriminación étnica, donde los afrodescendientes tienen mayor percepción de discriminación étnica que los mulatos y los mestizos, así como los mestizos tienen mayor percepción de discriminación étnica que los mulatos, siendo los blancos el grupo que presenta una menor percepción de la discriminación étnica. En cuanto a los efectos de dicha discriminación sobre la autoestima colectiva se observa que mulatos presentan mayores niveles de autoestima colectiva que afrodescendientes, blancos y mestizos, mientras que los blancos tienen mayores niveles de autoestima colectiva que los mestizos y por último los mestizos presentan mayores niveles de autoestima que los afrodescendientes.

Mientras en el caso de discriminación racial, solo se observan diferencias poblacionales de los "mestizos o nativos americanos", respecto de todos los grupos, donde los mestizos tienen mayor percepción de discriminación racial que los blancos, no obstante, estos presentan menos discriminación racial que los afrodescendientes y que los mulatos.

Finalmente se observa que los blancos presentan mayores niveles de autoestima colectiva que los mestizos, estos últimos, mayores niveles de $\mathrm{AC}$ que los afrodescendientes. Y por último los mulatos presentan mayores niveles de AC que los mestizos.

Las diferencias culturales en el autoconcepto también sugieren que la autoestima relativamente alta característica de los "negros" puede no caracterizar a otros grupos minoritarios raciales. Por ejemplo, tanto las culturas como los individuos dentro de las culturas difieren en su respaldo al individualismo, con su concepción de las personas como independientes entre sí y su enfoque en los objetivos personales, la singularidad personal y el control personal.

En muchos contextos culturales de América del Norte y Europa, es más probable que se vea a la persona como si tuviera un yo que sea estable y que trascienda las relaciones y las situaciones. En muchas otras culturas más colectivistas, es más probable que se considere que la persona tiene un yo que es flexible y depende del contexto (Fiske, Kitayama, Markus y Nisbett, 1998). Asociada con el individualismo y la autoconstrucción independiente está la tendencia a mantener y mejorar la autoestima, particularmente a través de los esfuerzos por destacar o ser superiores a los demás. Esta tendencia es más débil o incluso ausente en algunas culturas que enfatizan la autointerpretación interdependiente, en la cual la armonía en las relaciones sociales es un objetivo más importante (Fiske et al, 1998; Heine, Lehman, Markus y Kitayama, 1999; Markus y Kitayama, 1991). De hecho, Heine et al. (1999) argumentaron que en algunas culturas en las que el yo se interpreta como interdependiente, existe una práctica común y elaborada de autocrítica al servicio de la superación personal, que promueve la armonía en las relaciones (Kitayama, Markus, Matsumoto y Norasakkunkit, 1997). Estos autores argumentaron que esa práctica puede explicar los bajos niveles de autoestima que se encuentran en algunos países del este de Asia, como Japón. Las culturas que enfatizan el colectivismo también tienen con frecuencia una norma de modestia en las auto-presentaciones, lo que también contribuye a puntuaciones bajas en las medidas de autoestima (Tafarodi y Swann, 1996). Oyserman et al. (2002) revisaron estudios sobre la relación entre 
individualismo, colectivismo y autoestima e informaron que las diferencias individuales en el individualismo se correlacionan con las diferencias individuales en la autoestima, tanto en América del Norte como en Japón. El colectivismo, sin embargo, no se correlacionó consistentemente con la baja autoestima.

Si las diferencias culturales explican algunas de las diferencias en la autoestima, los efectos regionales deberían ser evidentes.

Dentro de las aportaciones de este estudio, se lograron visibilizar percepciones de conformidad y disconformidad que poseen los colombianos respecto de las prácticas habituales que se generan en el lugar en que residen, al igual que conocer cómo perciben su situación actual y la discriminación recibida.

Una de las principales limitaciones dentro de este estudio fue la extensión del cuestionario aplicado, puesto que la investigación forma parte de un proyecto en el que se incluyen cuestionarios adicionales a los utilizados, correspondiente a dos estudios más, generando cansancio y agotamiento mental en las personas encuestadas.

En estudios posteriores se pretende controlar los análisis por estado de salud, considerando que la autoestima parece relacionarse con la salud mental y también con la salud física (Hudd et al., 2000; Sánchez, Aparicio y Dresch, 2006). Se ha supuesto que las personas que presentan mejor ajuste y mejor estado de bienestar psicológico tendrán también una valoración positiva de su autoconcepto (Baumeister, 1998; Zacarés, Iborra, Tomás y Serra, 2009) por su nivel socio educativo, toda vez que al determinar el acceso a las oportunidades educativas y de empleo, la segregación ha sido un mecanismo clave mediante el cual se ha creado y reforzado la desigualdad racial (Williams, 2006).

De igual modo este estudio en general facilitará a otras investigaciones, contribuyendo con información en cuanto a la discriminación ejercida en colombianos y sus efectos sobre la autoestima individual y colectiva.

Se sugiere analizar el ajuste de este modelo en otros colectivos de migrantes o incluso de chilenos, con el objetivo de identificar posibles diferencias respecto a la discriminación ejercida y autoestimas entre un grupo y otro, así mismo las discriminaciones que se generan en la ciudad para identificar si es que la discriminación ejercida hacia los inmigrantes colombianos difiere de la que ejercen en la población chilena u otros grupos de migrantes.

\section{Referencias}

Abello, R., Amarís, M., Blanco, A., Madariaga, C., Díaz, D., Arciniégas, T. (2008) Autoestima, depresión y anomia en personas que no han sido víctimas de violencia política y social. Investigación \& Desarrollo, 16 (2), 214-231

Aguilar, M. (2014). Discriminaciones múltiples de los migrantes en perspectiva de derechos BARATARIA, Revista Castellano-Manchega de Ciencias Sociales, 17, 39-54.

Arenas, P., Urzúa, A., (2016). Estrategias de Aculturación e identidad étnica. Un estudio en migrantes Sur-Sur en el norte de Chile. Universitas Psychologica, 15(1), 117-128.

Basabe, N., Páez, D., Aierdi, X., \& Jiménez-Aristizábal, A. (2009). Salud e inmigración. Aculturación, bienestar subjetivo y calidad de vida. Zarautz, España: Ikuspegi.

Baumeister, R.F. (1998). The self. En D. T. Gilbert, S. T. Fiske y G. Lindzey (Eds.). The Handbook of Social Psychology, 4(1), 680-740. Bhugra, D. (2004). Migration and mental health. Acta Psychiatrica Scandinavica, 109 (4), 243-258.

Bourguignon, D., Seron, E., Yzerbyt, V. y Herman, G. (2006). Perceived group and personal discrimination: Differential effects on personal self-esteem. European Journal of Social Psychology, 36, 773-789.

Branscombe, N. R., Schmitt, M. T., \& Harvey, R. D. (1999). Perceiving pervasive discrimination among African Americans: Implications for group identification and well-being. Journal of Personality and Social Psychology, 77(1), 135-149. http://dx.doi.org/10.1037/00223514.77.1.135

Broman, C.L., Neighbors, H. W. \& Jackson, J. S. (1988). Racial group identification among Black adults. Social Forces, 67, $146-158$.

Brondolo, E., Kelly, K.P., Coakley, V., Gordon, T., Thompson, S., Levy, E., et al. (2005). The perceived ethnic discrimination questionnaire. J. Appl. Soc. Psychol. 35(2), 335-365.

Brondolo, E., Rieppi, R., Kelly, K.P., Gerin, W. (2003). Perceived racism and blood pressure. Ann. Behav. Med. 25 , 55-65.

Campal, F., (2014) Lucha contra la discriminación racial y étnica, aproximaciína los conceptos clave. Cuaderno didáctico 1. Accem. Disponible en http://www.gestionpolicialdiversidad.org/index.php/component/phocadownload/category/4-diversidad-etnica?download=89:2014-accem-cuaderno-didactico-lucha-contra-la-discriminacion-aproximacion-a-los-conceptos-clave 
Clark, R., Anderson, N.B., Clark, C.R., and Williams, D. R. (1999). Racism as a stressor for African Americans: A biopsychosocial model. Am. Psychol. 54, 805-816.

Cohen, J. (1988). Statistical Power Analysis for the Behavioral Sciences. Second Edition. Hillsdate, NJ: LEA.

Contrada, R.J., Ashmore, R.D., Gary, M.L., Coups, E., Egeth, J.D., Sewell, A., et al. (2001). Measures of ethnicity-related stress. J. Appl. Soc. Psychol. 31, 1775-1820.

Cooley, C.H. (1902). Human nature and the social order. New York: Schocken.

Crocker, J. y Major, B. (1989). Reactions to stigma: The moderating role of justification. En M. P. Zanna y J. Olson (Eds.), The psychology of prejudice: The Ontario Symposium (pp. 289-314). New Jersey: Lawrence Erlbaum.

Crocker, J., Voelk1, K., Testa, M., \& Major, B. (1991). Social stigma: The affective consequences of attributional ambiguity. Journal of Personality and Social Psychology, 60, 218-228.

De la Torre, R. M. (2011). La feminización de las remesas. La migración laboral hacia los Estados Unidos. En I. Solano. Migrantes somos y en el camino andamos: Ensayos sobre identidad, migración y cultura transfronteriza, (pp. 157-186). México, D.F.: Colección Miradas del Centauro

Dion, K.L. (1986). Responses to perceived discrimination and relative deprivation. Relative deprivation and social comparison: The Ontario Symposium, 4, 159-179.

Ellemers, N., Wilke, H., \& van Knippenberg, A. (1993). Effects of the legitimacy of low group or individual status on individual and collective status-enhancement strategies. Journal of Personality and Social Psychology, 64(5), 766-778. http://dx.doi.org/10.1037/00223514.64.5.766

Fiske, A.P., Kitayama, S., Markus, H. R., \& Nisbett, R.E. (1998). The cultural matrix of social psychology. In D. T. Gilbert \& S. T. Fiske (Eds.), The handbook of social psychology (Vol. 2, 4th ed., pp. 357-411). New York: McGraw-Hill.

Foster, M.D. (2000). Utilization of global attributions in recognizing and responding to gender discrimination among college women. Current Psychology, 19, 57-69.

Garrido, L.E., Abad, F.J., \& Ponsoda, V. (2011). Performance of Velicer's minimum average partial factor retention method with categorical variables. Educational and Psychological Measurement, 71(3), 551-570. http://dx.doi.org/10.1177/0013164410389489

Gómez-Lugo, M., Espada, J., Morales, A., Marchal-Bertrand, L., Soler, F., Vallejo-Medina, P. (2016). Adaptation, validation, Reliability and factorial equivalence of the Rosenberg Self-Esteem Scale in Colombian and Spanish population. The Spanish Journal of Psychology, 19: 1-12. Doi: 10.107/sjp.2016.67

Goodman, L.A. (1961). Snowball Sampling, Annals of Mathematical Statistics, 32, 148-170.

Guzmán, K., González, B., \& Rivera, M. (2014). Recursos psicológicos y percepción de la migración en menores con familiares migrantes. Revista Latinoamericana de Ciencias Sociales, Niñez y Juventud, 13 (2), 701-714.

Guzmán-Carrillo, K.Y. \& Rivera-Heredia, M.E. (2012). Siguiendo la pista de la migración. México, D.F.: Consejo Estatal de Ciencia, Tecnología e Innovación de Michoacán (Cecti).

Hansen, N. \& Sassenberg, K. (2006). Does social identification harm of serve as a buffer? The impact of social identification on anger after experiencing social discrimination. Personality and Social Psychology Bulletin, 32, 983-996.

Heine, S.J., Lehman, D. R., Markus, H.R., \& Kitayama, S. (1999). Is there a universal need for positive self-regard?. Psychological Review, 106, 766-795.

Heiss, J., \& Owens, S. (1972). Self-evaluations of Blacks and Whites. American Journal of Sociology, 78, 360-370

Huang, W., Chen, S., Pakpour, A. \& Lin, C. (2018). The Mediation Role of Self-Esteem for Self-Stigma on Quality of Life for People with Schizophrenia: A Retrospectively Longitudinal Study. Journal of Pacific Rim Psychology, 12, E10. doi:10.1017/prp.2017.18

Hudd, S., Dumlao, J., Erdmann-Sager, D., Murray, D., Phan, E., Soukas, N. y Ykozuka, N. (2000). Stress at college: effects on health habits, health status and self-esteem. Canadian Journal of Occupational Therapy, 67 (4),230-238.

INE (2017). Resultados censo 2017. Disponible en https://resultados.censo2017.cl/.

Jasinskaja-Lahti, I., Liebkind, K., Jaakkola, M., \& Reuter, A. (2006). Perceived Discrimination, Social Support Networks, and Psychological Well-being Among Three Immigrant Groups. Journal of Cross-Cultural Psychology, 37(3), 293-311. http://dx.doi. org/10.1177/0022022106286925

Jones, J. M. (1999). Cultural racism: The intersection of race and culture in intergroup conflict. In D. A. Prentice \& D. T. Miller (Eds.), Cultural divides: Understanding and overcoming group conflict (pp. 465-490). New York: Russell Sage Foundation.

Kernis, Mh., Granneman, B.D. y Mathis, L.C. (1991). Stability of self-esteem as a moderator of the relation between level of self-esteem and depression. Journal of Personality and Social Psychology, 61, 80-84

Kitayama, S., Markus, H. R., Matsumoto, H., \& Norasakkunkit, V. (1997). Individual and collective processes in the construction of the self: Self-enhancement in the United States and self-criticism in Japan. Journal of Personality and Social Psychology, 72, $1245-1267$. 
Kong, F., \& You, X. (2013). Loneliness and self-esteem as mediators between social support and life satisfaction in late adolescence. Social Indicators Research, 110(1), 271-279.

Kong, F., Zhao, J. \& You, X. (2012). Emotional intelligence and life satisfaction in Chinese university students: The mediating role of self-esteem and social support. Personality and Individual Differences, 53(8), 1039-1043.

Kong, F., Zhao, J. \& You, X. (2013). Self-Esteem as Mediator and Moderator of the Relationship Between Social Support and Subjective Well-Being Among Chinese University Students. Soc Indic Res. 112, 151. https://oi.org/10.1007/s11205-012-0044-6

Krieger, N., Smith, K., Naishadham, D., Hartman, C. \& Barbeau, E. (2005) Experiences of discrimination: Validity and reliability of a self-report measure for population health research on racism and health. Social Science \& Medicine, 61 (7), 1576-1596.

Lewis, T.T., Cogburn, C.D., \& Williams, D.R. (2015). Self-reported experiences of discrimination and health: scientific advances, ongoing controversies, and emerging issues. Annual Review of Clinical Psychology, 11, 407-440.

Li, J., Fang, M., Wang, W., Sun, G. and Cheng, Z., 2018. The Influence of Grit on Life Satisfaction: Self-Esteem as a Mediator. Psychologica Belgica, 58(1), 51-66. DOI: http://doi.org/10.5334/pb.400

Lönnqvist, J.-E., Hennig-Schmidt, H. y Walkowitz, G. (2015). Ethnicity and sex-based discrimination and the maintenance of selfesteem. PLoS ONE, 10(5), 2-19. http://dx.doi.org/10.1371/ journal pone.0124622

Lueck, K. \& Wilson, M. (2011). Acculturative stress in Latino Immigrants: The impact of social, socio-psychological and migration related factors. International Journal of Intercultural Relations (35), 186-195.

Luhtanen, R. \& Crocker, J. (1992). A collective self-esteem scale: self evaluation of one's social identity. Personality and social Psychology Bulletin, 18, 302-318.

Markus, H. R., \& Kitayama, S. (1991). Culture and the self: Implications for cognition, emotion, and motivation. Psychological Review, 98, pp. 224-253.

Mascie-Taylor, N. \& Little, M. (2004). History of Migration Studies in Biological Anthropology. American Journal of Human Biology, $16,356$.

McCarthy, J.D., \& Yancey, W.L. (1971). Uncle Tom and Mr. Charlie: Metaphysical pathos in the study of racism and personal disorganization. American Journal of Sociology, 76, pp. 648-672.

Major, B. (1994). From social inequality to personal entitlement: The role of social comparisons, legitimacy appraisals, and group membership. Advances in experimental social psychology, 26, 293-348.

Major, B., Sciacchitano, A.M. \& Crocker, J. (1993). In-group versus out-group comparisons and self-esteem. Personality and Social Psychology Bulletin, 19, 711-721.

Mckoy, S.K., \& Major, B. (2003). Group identification moderates emotional responses to perceived prejudice. Personality and Social Psychology Bulletin, 29, 1005-1017

Mead, G.H. (1934). Mind, self, and society. Chicago: University of Chicago Press.

Myer, I.H. (2003). Prejudice as stress: Conceptual and measurement problems. Am. J. Public Health, 93, 262-265.

Myers, L., \& Sirois, M. J. (2004). Spearman correlation coefficients, differences between. Encyclopedia of statistical sciences, 1-2. Doi: https://doi.org/10.1002/0471667196.ess5050.

Nylund, K.L., Asparouhov, T., Muthen, B. (2007). Deciding on the Number of Classes in Latent Class Analysis and Growth Mixture Modeling: A Monte Carlo Simulation Study. Structural Equation Modeling: A Multidisciplinary Journal, 14, 535-569. http://dx.doi.org/10.1080/10705510701575396

Noh, S. \& Kaspar, V. (2003). Perceived discrimination and depression: Moderating effects of coping, acculturation, and ethnic support. American Journal of Public Health, 93, 232, 238.

Organización Internacional para las Migraciones -OIM (2006). Derecho internacional sobre migración. Glosario sobre migración, $N^{\circ}$ 7, Suiza. Disponible en https://publications.iom.int/system/files/pdf/iml_7_sp.pdf

Organización Internacional para las Migraciones- OIM (2010). Informe sobre las migraciones en el mundo 2010. Disponible en http:// publications.iom.int/system/files/pdf/wmr_2010_spanish.pdf?language $=$ es

Organización de Naciones Unidas - ONU [Internet]. 2018.. Disponible en: http://www.un.org/es/sections/issues-depth/migration/index.html

Oyserman, D., Coon, H.M., \& Kemmelmeier, M. (2002). Rethinking individualism and collectivism: Evaluation of theoretical assumptions and meta-analyses. Psychological Bulletin, 128, 3-72.

Paradies, Y. (2006). A systematic review of empirical research on self-reported racism and health. International Journal of Epidemiology, 35, 888-901.

Pascoe, E. A., Richman, L. S. (2009). Perceived Discrimination and Health: A Meta-Analytic Review. Psychological bulletin, 135, 531-554. Pettigrew, T.F. (1967). Social evaluation theory: Convergences and application. In D. Levine (Ed.), Nebraska Symposium on Motivation,

15, pp. 241-311. Lincoln: University of Nebraska Press. 
Pinillos, M. (2012). Intervención psicosocial y educativa para prevención de riesgos asociados a procesos migratorios. Revista Latinoamericana de Ciencias Sociales, Niñez y Juventud, 1 (10), 579-591.

Porter, J.R. \& Washington, R.E. (1979). Black identity and self-esteem: A few of studies of Black self-concept, 1968-1978. Annual Review of Sociology, 5, 53-74.

Postmes, T., Branscombe, N., Spears, R., \& Young, H. (1999). Comparative processes in personal and group judgments: Resolving the discrepancy. Journal of Personality and Social Psychology, 76, 320-338

Roberts, J.E. y Monroe, S.M. (1992). Vulnerable self-esteem and depressive symptoms: prospective findings comparing three conceptualisations. Journal of Personality and Social Psychology, 62 (5), 804-812.

Rojas-Barahona, Cristian A, Zegers P, Beatriz, \& Förster M, Carla E. (2009). La escala de autoestima de Rosenberg: Validación para Chile en una muestra de jóvenes adultos, adultos y adultos mayores. Revista médica de Chile, 137(6), 791-800.

Rosenberg, M. (1965). Society and the adolescent self-image. NJ: Princeton University Press.

Ruggiero, K. M., \& Taylor, D.M. (1997). Why minority group members perceive or do not perceive the discrimination that confronts them: The role of self-esteem and perceived control. Journal of Personality and Social Psychology, 72, 373-389.

Rowley, S. J., Sellers, R. M., Chavous, T.M., \& Smith, M. A. (1998). The relationship between racial identity and self-esteem in African American college and high school students. Journal of Personality and Social Psychology, 74, 715-724.

Sánchez, M., Aparicio, M., \& Dresch, V. (2006). Ansiedad, autoestima y satisfacción autopercibida como predictores de la salud: diferencias entre hombres y mujeres. Psicothema, 18(3), 584-590.

Schreiber, J.B., Nora, A., Stage, F. K., Barlow, E. A., \& King, J. (2006). Reporting Structural Equation Modeling and Confirmatory Factor Analysis Results: A Review. Journal of Educational Research, 99, 323-338. https://doi.org/10.3200/JOER.99.6.323-338

Schmader, T., \& Major, B. (1999). Stigma and self-esteem: Situational construction of self-worth. Journal of Experimental Social Psychology, 35, 47-67

Schmitt, M.T., Branscombe, N.R., Postmes, T., Garcia, A. (2014). The consequences of perceived discrimination for psychological well-being: a meta-analytic review. Psychological bulletin, 140, 921-948.

Schmitt, M. T. \& Branscombe, N. R. (2002). The Meaning and Consequences of Perceived Discrimination in Disadvantaged and Privileged Social Groups. European Review of Social Psychology, 12, 167-199. Doi: 10.1080/14792772143000058

Skorek, M., Song, A.V., Dunham, Y. (2014). Self-Esteem as a Mediator between Personality Traits and Body Esteem: Path Analyses across Gender and Race/Ethnicity. PLOS ONE 9(11): e112086. https://doi.org/10.1371/journal.pone.0112086

Steele, C.M. (1997). A threat in the air: How stereotypes shape intellectual identity and performance. American Psychologist, 52, 613-629

Steele, C. M. \& Aronson, J. (1995). Stereotype threat and the intellectual test-performance of African-Americans. Journal of personality and social psychology, 69 (5), 797-811

Tafarodi, R.W., \& Swann, W.B. (1996). Individualism-collectivism and global self-esteem. Journal of Cross-Cultural Psychology,27, $651-672$.

Tajfel, H. y Turner, J.C. (1986) The social identity theory of intergroup behavior. En S. Worchel y W. Austin (Eds.) Psychology of Intergroup Relations (pp. 7-24). Chicago: Nelson Hall. (Existe versión castellana en J.F. Morales y C. Huici (Eds.) (1989) Lecturas de Psicología Social. Madrid. UNED. pp.225-259)

Tynes, B. M., Umaña-Taylor, A. J., Rose, C. A., Lin J., Anderson, C. J. (2012). Online racial discrimination and the protective function of ethnic identity and self-esteem for African American adolescents. Dev Psychol. 48(2), 343-355. doi: 10.1037/a0027032

Twenge, J.M. y Crocker, J. (2002). Race and self-esteem: Metaanalyses comparing Whites, Blacks, Hispanics, Asians, and American Indians and comment on Gray-Little and Hafdahl (2000). Psychological Bulletin, 128(3), 371-408. Disponible en: doi: http://dx.doi. org/10.1037/0033-2909.128.3.371

Urzúa, A., Basabe, N., Pizarro, J. J., Ferrer, R., (2017) Afrontamiento del estrés por aculturación: inmigrantes latinos en Chile. Universitas Psychologica, 16(5), 1-13. doi: 10.11144/Javeriana.upsy16-5.aeai

Urzúa, A., Boudon, S., Caqueo-Urízar, A. (2017). Salud Mental y Estrategias de Aculturación en inmigrantes colombianos y peruanos en el Norte de Chile. Acta Colombiana de Psicología, 20(1): 70-79. Doi: 10.14718/ACP.2017.20.1.5

Urzúa, A., Cabieses, B. (2018). Salud y Bienestar en población migrante en Chile: el aporte de los proyectos FONDECYT a la evidencia internacional. Cuadernos Médico Sociales, 58(4): 1-12.

Urzua, A., Cabrera, C., Calderón, C., Caqueo-Urizar. A., (2019). The mediating role of self-esteem on the relationship between perceived discrimination and mental health in South American immigrants in Chile. Psychiatry Research. 271: 187-194. Doi: 10.1016/j. psychres.2018.11.028

Urzúa M., A., Caqueo-urizar, A., Calderón, C., Rojas, L. (2017). ¿el apoyo social mediatiza el efecto negativo del estrés por aculturación en la salud? un estudio en colombianos y peruanos inmigrantes en el norte de chile. Interciencia, 42(12), 818-822. 
Urzúa, A., Delgado, E., Rojas, M. Caqueo-Urízar, A. (2017). Social Well Being among Colombian and Peruvian Immigrants in Northern Chile. Journal of Immigrant and Minority Health. 19(5), 1140-1147. DOI 10.1007/s10903-016-0416-0

Urzúa, A., Ferrer, R., Godoy, N., Leppes, F., Trujillo, C., Osorio, C., Caqueo-Urízar, A., (2018) The Mediating Effect of Self-esteem on the Relationship between Perceived Discrimination and Psychological Well-being in Immigrants. Plos One. 13(6): e198413. https:// doi.org/10.1371/journal.pone.0198413.

Urzúa, A., Ferrer, R., Canales, V., Nuñez, D., Ravanal, I., Tabilo B. (2017). The influence of acculturation strategies in quality of life by immigrants in Northern Chile. Quality of Life Research. 26(3): 717-726. Doi: 10.1007/s11136-016-1470-8

Urzúa, A., Heredia, O., Caqueo-Urízar, A. (2016). Salud mental y Estrés por aculturación en inmigrantes sudamericanos en el norte de Chile. Revista Médica de Chile. 144, 563-570

Urzúa, A., Vega, M., Jara, A., Trujillo, S., Muñoz, R. Caqueo-Urízar, A., (2015). Calidad de vida percibida en Inmigrantes Sudamericanos en el norte de Chile. Terapia Psicológica, 33(2), 139 - 156.

Valiente, R., Sandín, B., Chorot, P., Santed, M. y González de Rivera, J. (1996). Sucesos vitales mayores y estrés: Efectos psicopatológicos asociados al cambio por migración. Psiquis, 17, 211-230.

Vásquez, R. (2009). Impacto de las migraciones en Chile. Nuevos retos para el pediatra. ¿Estamos preparados?. Revista Chile Pediatria, 80 (2), 132.

Velásquez, V. (2016). Discriminación étnica y exclusión: Una investigación sobre las percepciones de las personas de nacionalidad boliviana en la ciudad de Comodoro Rivadavia 2013-2015. (tesis de grado). Universidad Nacional de la Patagonia San Juan Bosco, Argentina. Disponible en https://www.margen.org/tesis/discriminacion.pdf

Ward, C., Bochner, S., Furnham, A.. (2001). The Psychology of Culture Shock. Psychology Press: Routledge.

Walker, I. \& Mann, L. (1987). Unemployment, relative deprivation, and social protest. Personality and Social Psychology Bulletin, 13, 275-283.

Williams, D. (2006). Race, Socioeconomic Status, and Health The Added Effects of Racism and Discrimination. The New York Academy of Sciences, 2, 1248.

Williams, D.R., Mohammed, S.A. (2009). Discrimination and racial disparities in health: evidence and needed research. Journal of Behavioral Medicine, 32, 20-47.

Yáñez, S. y Cárdenas, M. (2010). Estrategias de aculturación, indicadores de salud mental y bienestar psicológico en un grupo de inmigrantes sudamericanos en Chile. Salud \& Sociedad, 1, 51-70

Zacarés, J.J., Iborra, A., Tomás, J.M. y Serra, E. (2009). El desarrollo de la identidad en la adolescencia y adultez emergente: Una comparación de la identidad global frente a la identidad en dominios específicos. Anales de Psicología, 25(2), 316-329.

Zarza, M. y Sobrino, M. (2007). Estrés de adaptación sociocultural en inmigrantes latinoamericanos en Estados Unidos vs. España: Una revisión bibliográfica. Anales de psicología, 23, 72-84.

Zlobina, A. (2004). Contactos interculturales y diferencias culturales: la inmigración y el choque cultural en la CAV y Europa. Revista Vasca de Sociología y Ciencia Política, 38, 183-206. 